\title{
MPEG-21 Integrated Cross Layer QoS Adaptation Architecture for Multimedia Applications in Future MANETs to Support Heterogeneous Network Environment
}

\author{
Arunkumar. B. R \\ Professor \& HOD, MCA Dept. \\ Sir M Visvesvaraya Institute of Technology \\ Bangalore, Karnataka, India.
}

\begin{abstract}
Different variety of multimedia-enabled end-user devices continues increase in number and expectation of ubiquitous customer irrespective their terminal capabilities in a heterogeneous environment offered numerous opportunities to quality of service improvements.

Potential computing expects MANETs to work with interoperability offering quality of service as professed by the user in a heterogeneous environment. This article presents architecture for state-of-the-art wireless multi-hop networks, and identifies research issues that need to be addressed for successful streaming with content awareness using protocol such as RTSP under heterogeneous environment for the next generation of wireless mobile ad hoc networks. The proposed architecture includes integration of MPEG-21 multimedia framework, digital content verification engine, cross layer design and interaction at various layers including network and MAC layers and IEEE 802.21 to handle mobility in MANETs to offer quality of service provisioning.
\end{abstract}

\section{General Terms}

Mobile ad hoc wireless networks, quality of service (QoS), MPEG-21, multimedia framework, cross-layer design, Ubiquitous computing.

\section{Keywords:}

IEEE 802.21 Architecture, Cross layer RTSP, Multimedia Applications, Mobility, Bandwidth estimation, Cross layer adaptation

\section{INTRODUCTION}

Wireless technology applications are of convenience, flexibility and popular. The ubiquitous mobile computing environment results in a heterogeneous environment where various wireless platforms such as cellular networks, WLANs, and MANETs integrate. Integration of different technologies with different capabilities and functionalities is an extremely complex task and involves issues at all layers of the protocol stack.

The multimedia on the network includes all kinds of media including text, image, 3D graphics, audio and video are produced, distributed, shared, managed and consumed on-line. The same end user may use various networks, like the Internet, Fiber, Wi-Fi, Wi-MAX, GPRS, 3G and so on and use different devices [1] [2][3].

A complete characterization of both the content, the usage context is highly needed to facilitate the selection and delivery of the content in the ubiquitous environment to offer the quality of service and satisfy ubiquitous end user. To satisfy these needs, MPEG-21 framework aims to provide solution. However, dealing with all these issues has resulted in complex solution. Due to the complexity of this standard, it is difficult to implement all MPEG-21 specifications in a single application, and therefore the integration of several tools, each implementing a specific functionality (e.g. adaptation, license processing, or streaming of the content, etc.) in the MPEG-21 framework, is necessary. MPEG-21 does not specify the technologies to be used and the manner of integrating the tools when developing MPEG-21 applications. This allows great flexibility for implementers [1] [2] [3].

Future computing expects MANETs to work with interoperability offering quality of service as perceived by the user in a heterogeneous environment. This article envisions architecture for state-of-the-art wireless multi-hop networks, and identifies research issues that need to be addressed for successful content aware streaming using protocol such as RTSP under heterogeneous environment for the next generation of wireless mobile ad hoc networks.

Mobile ad hoc wireless networks (MANETs) are multi-hop, selfcreating, self-organizing, self-administering, mobile, dynamictopology, infrastructure-less networks. In order to offer unique practical benefits and flexibility for certain environments and certain applications, several cross layer adaptations have to be integrated, coordinated and synchronized with routing, medium (or channel) access, mobility management, power management, security, and quality of service (QoS) issues etc. The lack of fixed infrastructure in ad hoc networks means that there must be mechanisms carefully designed to overcome numerous challenges efficiently. Otherwise, the user perceived QoS may put down [10] [11] [12].

QoS is the performance level of a service determined by the network to the user. The goal of QoS provisioning is to achieve a more guaranteed network behavior in terms of end user QoS perspective and network resources utilization. The most important QoS parameters for multimedia applications are delay, delay variance, available bandwidth, link stability, connectivity and so on [8][9][10][11][12]-[25].

QoS provisioning and multimedia transport involves devising novel solutions across various layers in the network protocol stack including application layer. To provide efficient QoS support we need adaptation of cross layer architecture which can coordinate to produce the best user-perceived quality. 
The cross layer adaptation facilitate protocols use the state information elegant throughout the stack to adapt their actions accordingly [8] [9] and to achieve substantial performance gain. Cross layer QoS adaptation is a new area of research, offers new challenges, and needs to be explored in depth.

Upper-layer transport protocols namely, Real-time Transport Protocol-RTP and Real Time Control Protocol-RTCP used for streaming applications, RTP which is designed to provide endto-end transportation and to support real-time applications works with

best effort delivery policy in an internet environment. There should be a mechanism to help regulating video streaming packets before or after the device, to estimate available bandwidth to decide on the packet sizes, to measure connectivity of the network, quality of service routing, resource reservation, congestion control mechanisms, mechanisms to reduce end-end delay and maximizing the video quality at receiving side, etc.

F. Bader et al in his work on "User-Centric Analysis of Perceived QoS in 4G IP Mobile/Wireless Networks" highlighted that" mapping of user requirements into system concepts is needed in order to design future $4 \mathrm{G}$ mobile/wireless communication systems that satisfy user expectations".

To assure the guarantee of QoS support at different layers as perceived by the user, the new MPEG-21 multimedia frame work defines several parts to facilitate Digital item consumption. This work proposes to design a novel architecture which integrates MPEG-21 with existing RTSP extended for future computing and adapt a suitable cross layer architecture which shares the bandwidth, connectivity and user information across physical, MAC, Link, Network, Transport and application layer. The cross layer adaptation and MPEG-21 integration with RTSP are to ensure quality of routing and streaming. Further, this proposed comprehensive design of the multimedia player is a complex system involving networking, media and user interactions management, Cyber law enforcement to ensure the legal issues, etc.

\subsection{Moving Picture Experts Group (MPEG) Multimedia Framework}

MPEG is a working group of ISO which has developed international standards for compression, decompression, processing and coded representation of moving pictures, audio, and their combination, in order to satisfy a wide variety of applications such as Video CD, MP3, DVD, Satellite TV, digital cable, HDTV, Video on demand and PC video streaming, etc [7][8][9].

The MPEG family consists of MPEG-1 and MPEG-2 provide interoperable ways of representing audiovisual content, commonly used on digital media and on the air, MPEG-4 defines how to represent content, MPEG-7 specifies how to describe content.MPEG-21 provides a truly interoperable multimedia framework which define the technology needed to support Users to exchange, access, consume, trade and otherwise manipulate Digital Items in an efficient, transparent and interoperable way.

MPEG-21 is a next generation multimedia framework define to support transactions that are interoperable and highly automated, particularly focusing on digital rights management (DRM) requirements, multimedia access and delivery across a wide range of networks and devices[7][8][9].

\section{RELATED WORK}

In recent relevant works of many years, various solutions addressing the issues of cross-layer optimization are proposed. Nevertheless, the mainstream of the papers limits investigations to wireless communication and cross interactions between the MAC and PHY layers. Most of the cross layer adaptations have not focused on user perceived QoS, user characteristics and legal issues, for example, [1]-[26] excluding [9] and [11]

The subsequent generation fixed and fixed-wireless networks have presented broadband access from home and office. The next generation mobile networks such as EGPRS and UMTS have abilities to offer higher throughputs, lower cost-per-bit of information and more "friendliness" to IP traffic [1][2][3].

Both mobile and stationary users of Wireless networks are still prone to some common drawbacks especially when networks are expected to work with interoperability in a heterogeneous environment. In terms of bandwidth, reliability and receiver device characteristics, wireless networks are heterogeneous. In wireless channels, packets can be delayed (due to queuing, propagation, transmission, and processing delays), lost or discarded due to complexity/resource limitations or display capabilities of the receiver. Hence, it is difficult to have the determined network behavior [1] [2] [3] [5] [11]

The inconsistent wireless network behavior and restricted network resources may not provide high bandwidth and do not satisfy stringent delay constraints resulting in unsatisfied end users. In literature analysis it can be noted that most of the works do not consider the interaction of user/application-level QoS and its mapping to network-level QoS. However, only a few papers have mapped the QoS expectations, for example, the work of Ahmed Toufik and Djama Ismail in [5] and [11]

The work of A. Flavio et al proposes a cross-layer standardcompliant scheduler for the uplink traffic in IEEE 802.16e networks which takes into account the link adaptation by the mobile stations [3].

An overview of both video streaming methodology and bandwidth estimation tools are given, and bandwidth adaptive video streaming architecture is introduced by T. Arsan in [4]

In [5], a seamless cross-layer inter-working between broadcasting network (i.e. DVB-T) and 802.11 WLAN network for adaptive and interactive mobile TV service delivery is proposed. The work uses a specific Adaptation Gateway (AG) at each Access Network to perform media adaptations. AG features a joint channel and video bit-rate awareness to tackle link degradations (signal strength quality, packet loss, etc.). Performance evaluations using an experimental test-bed are conducted and showed that the proposed cross-layer adaptation gateway reduces considerably packet losses and enhances the perceived quality of the TV service. By independently adapting each individual service, it is possible to address network and terminal heterogeneity and to offer interactive and personalized service to the end users.

The work of Qian Zhang et al presents a framework, which provides QoS support, for multimedia delivery over wireless Internet, across different layers. To provide efficient QoS support for different types of media over the best-effort networks, the work proposed a cross-layer architecture, which combines the application-level, transport-layer, as well as linklayer controls [6]. 
Eric Setton et al explored a cross-layer design framework for real-time video streaming, which maintains a general layered structure and identifies the key parameters to be exchanged between adjacent layers. In this context adaptive link layer techniques that adjust packet size, symbol rate, and constellation size according to channel conditions are used to improve link throughput, which in turn improves the achievable capacity region of the network. At the MAC and network layers, joint allocation of capacity and flow optimize the supportable traffic rate significantly, and consequently can improve the end to- end video quality by a wide margin [9].

It is worth to note that most of the related works on cross layer designs for QoS provisioning are based on IEEE 802.11 at data link layer. It is needless to say that one can use the recent IEEE 802.21 architecture which can support MANET for mobility. The next section provides the overview of the IEEE 802.21.

\section{MobiLity}

In the near future nodes in a MANETs may have to work with content distribution networks such as WLAN (IEEE 802.11x), GPRS (EDGE), 3G (UMTS, HSDPA), Wi-MAX (IEEE 802.16), MBWA (IEEE 802.20) to offer rich networking environment supporting multimedia applications. A node may have to support multihoming as a natural feature as explained in [1] and shown in Figure 1.

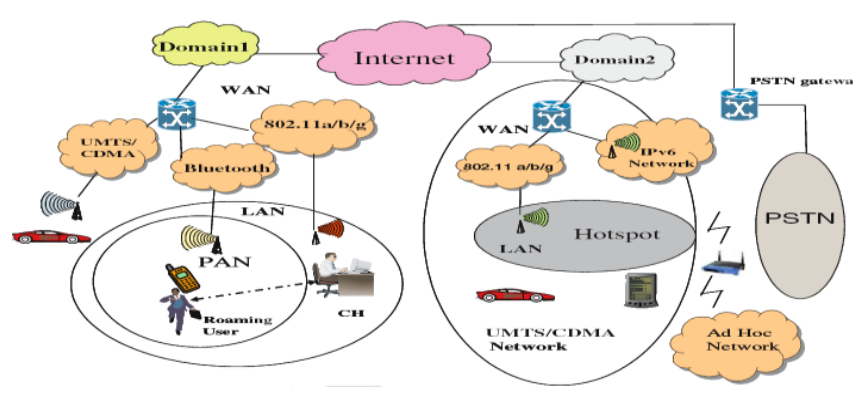

Figure1. A MANET to support in a heterogeneous environment [27].

To offer QoS in heterogeneous environment the IEEE standard can address and enable handover and interoperability between heterogeneous network types, including both 802 and non-802 networks. The 802.21 standard defines an abstraction layer, providing Media Independent Handover (MIH) functions with the goal of simplifying the management of handovers to and from different access technologies. In heterogeneous environment, mobile protocols such as Mobile IP, NEMO and MANET may benefit from the IEEE 802.21 standard [1] [2]. The architecture of IEEE 802.21 is shown in the Figure 2.

The IEEE 802.21 architecture adaptation may yield the following benefits as mentioned in [1], [2]:

a) Seamless co-existence of all the wireless technologies is required, including seamless mobility.

b) To optimize mobility and multi-homing cross layer design is a suitable approach. This includes cross-layer issues between transport, network, MAC, and even physical layers. For seamless integration of networks some bridging functions at MAC level are required and routing/MAC cross-layer design may help efficient and reliable routing.

c) Addressing multi-hop communications Management. d) Auto Self-configuration connectivity.

e) Bandwidth balancing in multi-homed devices amounts to deciding on which interfaces and paths to be used.

f) The available bandwidth estimation.

One can note that it is highly essential to integrate MPEG-21 with existing RTSP and adapt a suitable cross layer architecture which shares the bandwidth, connectivity and user information across physical, MAC, Link, Network, Transport and application layer and bring interoperability.

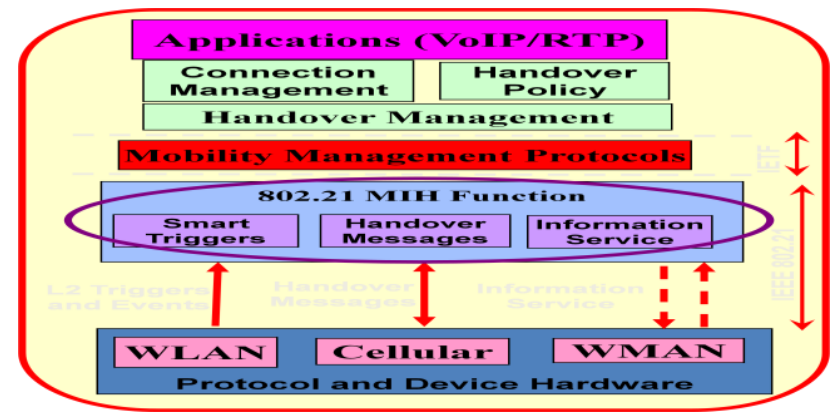

Figure 2: The architecture of IEEE 802.21 [27].

\section{CROSS LAYER ADAPTATION FOR RICH QOS}

Some of the QoS parameters e end-to-end delay, available bandwidth, probability of packet loss, connectivity, ensured legal activities with respect to the digital contents use and so on. Naturally, all the expected network resources must be available during the service invocation to credit the guarantee. The prime function is to find an appropriate path between the source and destination(s) that satisfies the essential resources. In this proposed architecture, we compute QoS multicast path based on connectivity index and bandwidth requirement parameters [20], [22] and [24].

Offering sustainable end-to-end quality through the full session as indicated by the user and ensuring all the activities are legal is another challenge. In delivering the multimedia content from the provider to the consumer many legal issues and actors are involved, interoperability plays a crucial role, digital content needs to be adapted to various transmission channels and terminal devices for delivery and hence calls for a multimedia framework. An ample framework that deals with all these issues for the entire delivery chain is MPEG-21 [1], [2]. MPEG-21 expects the network not only content distributed but more importantly content aware also. It offers/enables

- Content and service providers to describe their contents/services in an interoperable way.

- Means for describing and capturing the user profile and preferences.

- Network providers and device manufactures to describe their conditions and capabilities (i.e., context)

- Service providers to configure/personalize/ customize accordingly their services.

- Description formats for managing in heterogeneous environment. 
- $\quad$ Finally, MPEG-21 provides support for Digital Rights Management (DRM) which can be used for authentication, authorization, and accounting (AAA).

\subsection{MPEG-21 Digital Item Adaptation}

According to various transmission channels and terminal devices for delivery digital content need to be adapted. Digital Item Adaptation (DIA) can be achieved by applying various approaches such as adaptation at the server side, at the intermediate proxy or at the terminal.

We focus here only on the relevant requirements in terms of adaptation of user perception of quality and digital content management [2]. It includes explanatory information related to user characteristics, (e.g., user information and user preferences), terminal capabilities (e.g., codec capabilities and display capabilities), network characteristics (e.g., available bandwidth, delay, and error), and natural environment characteristics (e.g., location and time).

\subsubsection{User/application level QoS description using MPEG-21 meta-data}

To describe session parameters for announcements and invitations and to describe the capabilities of a system and possibly provide a choice between a numbers of alternatives, session description protocol is used.

The several flaws in the SDP [11] are recognized and it can be noted that MPEG-21 can fill the SDP gaps by providing metadata related to the user, terminal, content and network characteristics. We believe that building SDP on top of MPEG21 will give intelligent solutions to enable multimedia content access under a wide range of delivery conditions and usage environments. Presently IETF work presents a practical approach for harmonizing MPEG-21 with SDPng (GuenkovaLuy et al., 2005). Based on MPEG-21 tools and meta-data information, this work proposes a cross-layer architecture adaptation. The architecture is designed based on user perceived QoS, application QoS and network QoS. This system supports universal media access anywhere, anytime, using any type of terminal.

\section{CROSS-LAYER ADAPTATION}

Fig. 3 shows cross layer adaptation (CLA) architecture with a subset of protocols and possible cross-layer interactions between different layers. User specifications are described in MPEG-21 meta-data based on XML data format forming an important layer that should be also considered in the system. For example [11], terminal 8-bit color scheme support without loss of generality, let us take a terminal that supports 8-bit color scheme and supported frame rate of the terminal, etc could be expressed by an MPEG-21 XML element. These elements are carried by the SDPng description protocol at the connection phase. The streaming server uses the SDPng description to map the UED to the application layer.
The application layer uses the feedbacks to adapt the stream to dynamic network conditions. For example the adaptation is based on the whole changing values in UED (screen format, battery depletion level, user preferences, etc). At the network layer, an advanced interaction with upper layer and lower layer is necessary to assure QoS. If connectivity exists then only it is possible and easy to estimate available bandwidth and link stability [Cheikh Sarr et al," Bandwidth estimation for Ad hoc networks", IEEE transactions, Vol. X, April 2007]. The network layer based on the feedback of MAC layer computes multicast paths based on connectivity index and bandwidth availability or link stability.

An MPEG-21 driven QoS RTSP is used to map the application level QoS to network level QoS. At network layer routing path is computed based on connectivity index with application required bandwidth constraint.

For dynamic adaptation [11] the dynamic characteristics such as network load, IP Packet Delay Variation (IPDV), One-way Packet Loss (OWPL), Round-trip Delay (RTD) or network congestion level such as Random Early Detection (RED), Early Packet Discard (EPD), Early Congestion Notification (ECN), and ICMP source quench message need to be considered.

It is clear that allowing universal media access cannot be performed without taking into account dynamic conditions such as dynamic UED and mobility etc. To adopt these dynamic conditions MPEG-21 frame work and IEEE 802.21 implementations are proposed in this extended architecture.

\subsection{Multimedia Rights Management}

Future multimedia delivery platforms of the MANETs are necessarily to be intertwined with Multimedia Rights Management, also known as Digital Rights Management (DRM) or Content Protection (CP). Effective management of multimedia rights and licensing the rights to processing and consumption of multimedia contents is a challenge and can bring lawful control and revenue to the multimedia Industry. To support this many protocols, architectures, applications and wide range of business models should have to implement DRM and content protection. An adaptable multimedia delivery platform rights management framework needs to be designed which takes into account a wide variety of content distribution services and content source types [1] [2]. For end-to-end content protection and digital rights management with QoS management, there are several international and industry standards which are interoperable namely MPEG-21 IPMP, OMA and DMP (the Digital Media Project).

The present DRM standards have started war against the digital piracy implementing various technologies for rights management, concentrating on user, content authentication and enforced license rules. The dilemma is that current standards are limited in scope, inflexible and may, diminish the potential of digital media. They cause prices to increase and accessibility to diminish. Furthermore, the technologies have attempted to protect the delivery channels rather than the content. 


\section{CONCLUSION}
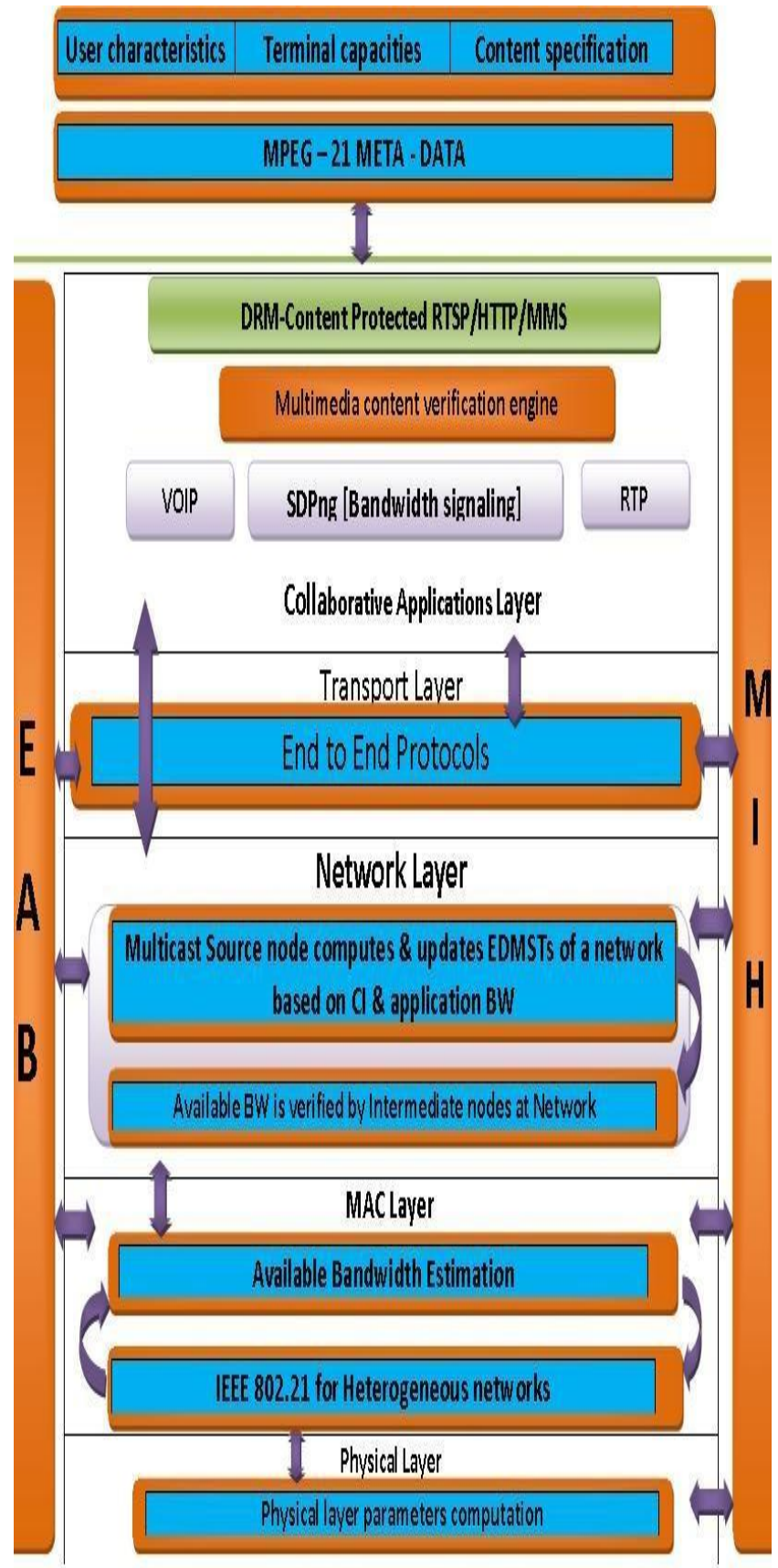

Figure 3: MPEG-21 CLA Architecture for Multimedia Applications in MANETs

This paper highlights the need, benefits of the cross-layer adaptation and design proposal of the protocol architecture using MPEG-21 framework, protocols such as RTSP, SDPng, etc at application layer, adaptation of cross layer across physical, network and application layer for QoS routing and mobility management using IEEE 802.21 architecture for heterogeneous environment. The unique characteristics of wireless ad hoc networks call for new design concept. The concept of cross-layer design sounds convincingly appealing. However, the adoption of a cross-layer is desirable and complex.

Introducing MPEG-21 as a new layer will add to further complexity of the system. Designing MANET to interoperable with other networks is challenging task. We expect that the most significant gains will come from interaction between different layers. Trade off between performance gains and design complexity should be balanced. It is highly important to keep the abstraction of layering while allowing information exchange between adjacent layers.

Most of the work in this research field is simulation based and it is hard to find countable number cross-layer proposals that have been tested comprehensively under real world traffic scenarios and hence QoS, power consumption and scalability of these approaches are not deterministic. However, in future computing need to be ubiquitous and networks should be content aware and distribution aware.

The future works need to design the multimedia content authentication engine which rightly manages the rights protects the contents, favor content creators and users and at the same time keep the potential of digital use high with affordable cost. The technologies have failed and continue to fail to curb the copyright violations if challenges are not turned into opportunities. The future MANETs need to be content-aware network. All the applications/ protocols need to be aware of the contents that they are using including streaming protocol like RTSP in application layer and cross layer routing protocols, etc.

\section{ACKNOWLEDGMENTS}

Dr.ARUN KUMAR B.R has been awarded the degree, Ph.D in Computer Science from DRAVIDIAN UNIVERSITY, a State University of Andrapradesh Govt., recognized by UGC, established in the year 1997, for his Thesis entitled, "Cross Layer Design for Quality of Service Multicasting in Mobile Ad-hoc Networks", with effect from 20-9-2012. He carried out his research work under the guidance of Dr.Lokanatha.C Reddy, Professor, CS dept., Dravidian University and Dr.Prakash.S Hiremath, Professor, CS dept., Gulbarga University. He has been awarded with three post graduate degrees, MCA, M.Phil (CS) M.Tech (CS\& E) from Kuvempu University, M.S University and from Dr.MGR Educational and Research institute University respectively. He obtained his post graduate diploma in IPR from National Law School of India University, Bangalore in July 2012. The author has published 19 research papers in National/International Journals. He has presented and published 11 papers in the National/International Conferences/proceedings including IEEE international conferences in India and abroad, wishes to place on record his sincere thanks to all those who helped in bringing out this paper. The author acknowledges the curtsey of the source, https://mentor.ieee.org/802.11/file/07/11-07-0453-00-0000-80221-midweek-plenary-update.ppt, for using the Figure 1 and Figure 2.

\section{REFERENCES}

[1] F. Bader et al, "User-Centric Analysis of Perceived QoS in 4G IP Mobile/Wireless Networks", CTR, King's College London, UK. Media Delivery Platforms Cluster report," Multimedia Delivery in the Future Internet, A Converged Network Perspective, published in October 2008.

[2] Giorgiana Ciobanu, Maria Teresa Andrad e , Pedro Carvalho, Eurico

Carrapatoso "An MPEG-21 Web Peer for the consumption of Digital Items" INESC Porto -378, 4200 - 465 
[3] Flavio A. Kubota, Juliana F. Borin and Nelson L. S. da Fonseca," Opportunistic Cross-layer Uplink Scheduler for the IEEE 802.16 standard", IEEE Communications Society publication in the IEEE ICC 2011 proceedings, 978-161284-231-8/11/2011, IEEE.

[4] T. Arsan, Kadir University, Computer Engineering Department, Turke "An Integrated Software Architecture for Bandwidth Adaptive Video Streaming", International Journal of Computer Science and Engineering Volume 2 Number 2.

[5] Ismail Djama and Toufik Ahmed," A Cross-Layer Interworking of DVB-T and WLAN for Mobile IPTV Service Delivery", IEEE TRANSACTIONS ON BROADCASTING, VOL. 53, NO. 1, MARCH 2007.

[6] Qian Zhang, Fan Yang, Wenwu Zhu," Cross-Layer QoS Support for Multimedia Delivery over Wireless Internet", EURASIP Journal on Applied Signal Processing 2005:2, 207-219, 2005 Hindawi Publishing Corporation.

[7] Jan Bormans, Jean Gelissen, and Andrew Perkis," MPEG21- The 21st century Multimedia Farmework”, pp. 53-62, IEEE SIGNAL PROCESSING MAGAZINE, March 2003.

[8] Marco Conti et al, "Cross-Layering in Mobile Ad Hoc Network Design", pp. 48-51, Published by the IEEE Computer Society, 2204

[9] Eric Setton, et al," CROSS-LAYER DESIGN OF AD HOC NETWORKS FOR REAL-TIME VIDEO STREAMING", pp. 59-66, IEEE Wireless Communications • August 2005.

[10] Satyabrata Chakrabarti, Lucent Technologies Amitabh Mishra, " QoS Issues in Ad Hoc Wireless Networks", pp. 142-148, IEEE Communications Magazine - February 2001

[11] AHMED Toufik, DJAMA Ismail," Delivering audiovisual content with MPEG-21-enabled cross-layer QoS adaptation", Journal of Zhejiang University SCIENCE A ISSN 1009-3095 (Print); ISSN 1862-1775 (Online), Ahmed et 784 al. / J Zhejiang Univ SCIENCE A 2006 7(5):784793.

[12] Arun Kumar B. R., Lokanatha C. Reddy and Prakash.S.Hiremath, "Mobile Ad Hoc Networks: Issues, Research Trends and Experiments", International Engineering \& Technology (IETECH) Journal of Communication Techniques, Vol. 2, No. 2, 057-063, AprilJune 2008.

[13] Arun Kumar B. R., Lokanatha C. Reddy and Prakash.S.Hiremath, "A Survey of Mobile Ad Hoc Network Routing Protocols", Journal of Intelligent System Research, Vol.2,No.1, pp.5-20, January-June 2008.

[14] Arun Kumar B. R., Lokanatha C. Reddy and Prakash.S.Hiremath, "Performance Comparison of Wireless Mobile Ad-Hoc Network Routing Protocols", IJCSNS International Journal of Computer Science and Network Security, Vol.8, No. 6, pp.337-343, June 2008.

[15] Arun Kumar B. R., Lokanatha C. Reddy and Prakash S.Hiremath, "High performance Modified DSR with Power Consumption Optimization for Mobile Ad hoc Networks", IJCSNS International Journal of Computer Science and Network Security, Vol.7, No. 5, pp. 169-178, May 2007.
[16] Arun Kumar B. R., Lokanatha C. Reddy, Prakash.S.Hiremath and Naresh.S.S, “ RTSP Audio and Video Streaming for QoS in Wireless Mobile Devices", IJCSNS International Journal of Computer Science and Network Security, Vol.8, No. 1, pp. 96-101, January 2008.

[17] Arun Kumar B. R., Lokanatha C. Reddy and Prakash.S.Hiremath, "Quality of Service in Mobile Ad Hoc Networks", Journal of Intelligent System Research, Vol.2, No.1, pp.21-39, January-June 2008.

[18] Arun Kumar B. R., Lokanatha C. Reddy and Prakash.S.Hiremath, "Cross Layer Design in Wireless Ad Hoc Networks", Journal of Analysis and Computing, Vol.4, No.2, pp.121-128, July-December 2008.

[19] Arun Kumar B. R., Lokanatha C. Reddy and Prakash.S.Hiremath, "Cross Layer Design for QoS Multicasting and Real Time Streaming in Mobile Ad Hoc Networks", Journal of Analysis and Computing, Vol.4, No.2, pp.129-134, July-December 2008.

[20] Arun Kumar B. R, Lokanatha C. Reddy, Rajan and Prakash S.Hiremath, "Route Failure Tolerant Multicast In Mobile Ad Hoc Networks Using Disjoint Minimum Spanning Trees", the second IEEE ICCSIT 2009, August 8 - 11, 2009, Beijing, China.

[21] Arun Kumar B. R, Lokanatha C. Reddy, Prakash S.Hiremath and Rajeswari Sheshadri, "K-Nodes Multicasting Minimum Cost Spanning Trees In Wireless Mobile", International Journal of Computer Applications [IJCA],www.ijcaonline.org/archives/number4/110-225.

[22] Arun Kumar B. R, Lokanatha C. Reddy and Prakash S.Hiremath, "Route Failure Tolerant Multicast in Mobile Ad Hoc Networks Using Disjoint Minimum Spanning Trees Based on Connectivity Index with Bandwidth constraint", the proceedings of $2^{\text {nd }}$ IEEE International Conference on Advance Computing, 19$20^{\text {th }}$,Feb.,2010,Tharpur University, Patiala, Punjab.

[23] Arun Kumar B. R, Lokanatha C. Reddy and Prakash S.Hiremath, "Cross Layer Design for Quality of Service Multicasting in MANETs", IETECH Journal of Information Systems, Vol.2, No.4, pp.185-192, 2008.

[24] Arun Kumar B. R, Lokanatha C. Reddy and Prakash S Hiremath, "Analysis of K-Connected MANETS for QoS Multicasting Using Edge Disjoint Spanning Trees Based On Connectivity Index", International Journal of Computer Applications, Vol. 3, No.12, pp.15-22, 2010.

[25] Arun Kumar B. R, Lokanatha C. Reddy and Prakash S Hiremath, "Cross-Layer Design for Quality of Service Multicasting in Mobile Ad Hoc Networks", Journal of System Management, Vol. 9, No. 3, pp. 21-32, 2011

[26] Arun Kumar B. R, "Issues of cyber laws and IPR in software Industry and software process Model", International Journal of Computer Applications (IJCA), (0975 - 8887) (Foundation of Computer Science), Volume 44- No16, April 2012 Harvard Press, APRIL 2012.

[27] https://mentor.ieee.org/802.11/file/07/11-07-0453-00-0000802-21-midweek-plenary-update.ppt 